\title{
Performance Assessment of Hybrid Parallelism for Large-Scale Reservoir Simulation on Multi- and Many-core Architectures
}

\author{
Amani AlOnazi \\ Extreme Computing Research Center \\ King Abdullah University of Science and Technology \\ Thuwal, Saudi Arabia \\ amani.alonazi@kaust.edu.sa
}

\author{
Marcin Rogowski \\ Ahmed Al-Zawawi \\ EXPEC Advanced Research Center EXPEC Advanced Research Center \\ Saudi Aramco \\ Dhahran, Saudi Arabia \\ marcin.rogowski@aramco.com \\ Saudi Aramco \\ Dhahran, Saudi Arabia \\ ahmed.zawawi.1@aramco.com
}

\author{
David Keyes \\ Extreme Computing Research Center \\ King Abdullah University of Science and Technology \\ Thuwal, Saudi Arabia \\ david.keyes@kaust.edu.sa
}

\begin{abstract}
Two trends are reshaping the landscape of petroleum reservoir simulators, one architecturally and one application driven: an increasing number of cores per node and increasing computational intensity arising from higher fidelity physics at each cell. Implicit algebraic solvers being the dominant kernels, we present hybrid MPI and OpenMP implementations of the linear solver of GigaPOWERS, a full-scale real-world massively parallel simulator for black oil and composition models. We also evaluate the impact of explicit communication and computation overlap by including the halo exchange in the task-dependency graph. We analyze the performance of these modifications across multi- and many-core architectures, i.e., Intel Haswell, Skylake, and Knights Landing, using a variety of synthetic and real-world models. The hybrid approach results in up to $50 \%$ reduction of time to solution on a 16 million-cell SPE10-like model on Skylake whereas on a smaller, 1 million-cell, model on Haswell and Knights Landing both implementations achieve very similar performance. In the real-world reservoir simulations, the hybrid parallelism has reduced communication volume, memory consumption, and improved load balancing.
\end{abstract}

Index Terms-Reservoir Simulation, Linear solver, Hybrid MPI-OpenMP, Task-based halo exchange, Communicationcomputation overlap, Many-core, Multi-core

\section{INTRODUCTION}

Supercomputers are increasingly essential to model conventional and unconventional reservoirs in the oil and gas industry. However, the development of scalable reservoir simulators has been complicated by architectural trends in supercomputing. Strong scalability within the node becomes more challenging as the number of cores per node increases and memory per core decreases. The latter factor has a significant impact on the parallel performance of memory-bound applications as the available memory bandwidth is shared and limited. Using shared memory parallelization in conjunction with MPI is commonplace to overcome some of these new limitations on today's petascale systems. This hybrid approach has the following advantages: avoiding message passing within a node, avoiding memory copies required in MPI-everywhere implementations, and load balancing without explicit data movement [1].

Reservoir simulations of heterogeneous data and complex geometry lead to complex nonlinear partial differential equations (PDEs). The system resulting from the finite-volume discretization in space and a fully implicit scheme in time of these PDEs is solved with an iterative Newton solver. At each Newton step, a preconditioned iterative solver is employed on the large, sparse and unstructured systems. In 2008, Fung et al. [2] introduced a parallel iterative method that uses a preconditioner to accelerate Krylov solver for the linear system within the constrained pressure residual (CPR) framework developed by Wallis [3]. CPR restricts the full system residual to the pressure part (which has been shown to affect the convergence most negatively by Behie and Vinsome [4]) and solves it approximately, then, applies the pressure solution to constrain the residual on the full system. Its robustness and efficiency have been demonstrated in many industry-standard reservoir simulators such as Schlumberger's ECLIPSE [5] and Saudi Aramco's GigaPOWERS [6]. GigaPOWERS is the first simulator capable of handling over billion-cell models and its code has been used a baseline for this paper.

There have been several attempts to investigate hybrid parallelizations and utilizing accelerators in reservoir modeling. While many solutions have been proposed so far to employ shared memory parallization in linear solvers [7]-[10], in this work, we focus on studying the performance of hybridization within reservoir simulator frameworks. In 2012, Dzyuba et al. [11] reported a study of hybrid MPI with multithreading and MPI with GPU computing of giant reservoir simulation. In 2014, Jaure et al. [12] discussed a prototyping platform 
for reservoir simulation with distributed memory parallelism in addition to a level of shared memory multithreading. In 2014, Fung et al. [13] presented hybrid parallelism and a GPU acceleration study of the GigaPOWERS pressure solver. In 2015, Liu et al. [14] introduced a platform for hybrid MPI and OpenMP parallel reservoir simulations on structured grids. In this work, we develop hybrid parallelization of the preconditioner and the sparse matrix-vector (SpMV) multiplication in GigaPOWERS that typically take around $80 \%$ of the total simulation time. These code optimizations are aimed at reducing the time to solution of reservoir simulator on multi- and many-core Intel architectures. While many models have been studied for intranode parallelization, in this implementation we select hybrid MPI+OpenMP because of its portability. The main contributions of this paper are:

- Employ shared memory parallelization of GigaPOWERS's preconditioner and SpMV kernels, as complementary to the distributed memory MPI model. Based on application knowledge, we reformulate the base preconditioner into a set of independent systems to be solved concurrently.

- Ensure communication-computation overlap in the SpMV kernel by taskification and including MPI calls in the task dependency graph for the asynchronous progress of nonblocking communication while computing locally.

- Present a comprehensive performance assessment of this approach on state-of-the-art many-core and multi-core Intel architectures using a variety of synthetic and realworld reservoir models.

\section{Reservoir Simulation Solver Formulation}

The computational domain is decomposed into a number of subdomains, each representing a finite volume of the reservoir containing multiphase, multicomponent fluids. The Jacobian matrix $A$ for each of the reservoir subdomains is decomposed as in Equation 1:

$$
A=P+E
$$

where matrix $P$ is block-tridiagonal with grid cells ordered first in the $Z$-direction of the $3 D$ reservoir mesh. $E$ contains the remaining non-zero data blocks not included in $P$. Assuming that $P$ is dominant, an approximate inverse preconditioner for $A$ is [2]:

$$
A^{-1} \approx M_{N}^{-1}=\left[I+\sum_{k=1}^{N}(-1)^{k}\left(P^{-1} E\right)^{k}\right] P^{-1}
$$

where the order of expansion, $N$, is typically 1 , and the action of $P^{-1}$ is computed by first generating a $L U$ factorization in a setup step and the forward/backward substitution step. The CPR method is constructed in a way that uses Equation 2 as the base-preconditioning method. Fung [2] referred to it as a special instance of the general line-solve power-series (LSPS). The construction of $P$ and $E$ is discussed in the work of Fung and Dogru [2]. The main point of CPR is solving the pressure system first and then the full system using a corrected residual based on the pressure update. The

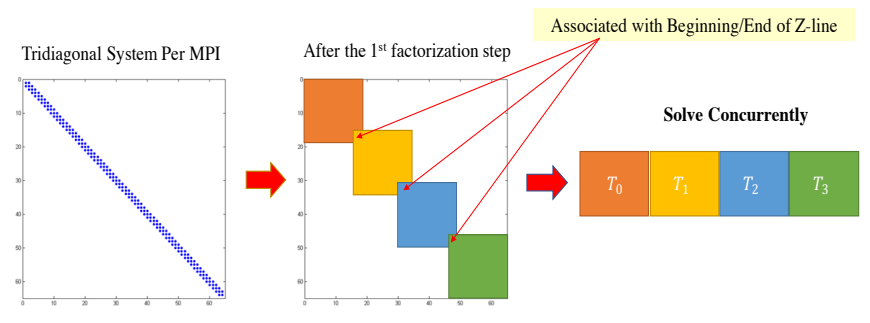

Fig. 1. An illustration of decomposing the matrix for the tridiagonal solve into independent set of tasks that are solved concurrently.

most time-consuming kernels in this linear solver are the preconditioning of the pressure and full systems within CPR, and the SpMV. The preconditioning of the system is the LSPS, with its tridiagonal downwards/upwards sweeps.

\section{INDEPENDENT TASKS FOR HYBRIDIZATION}

The preconditioner of the linear system is based on the linesolve LSPS method. The dominant matrix $P$ is constructed by assembling the linear equations associated with the cells of maximum transmissibility traces in the horizontal $X Y$ plane plus the vertical-transmissibility terms of each $Z$-line. This setup of the domain generates a set of $Z$-line systems that can be solved independently to construct the base preconditioner system. Based on this observation, the matrix is decomposed into a set of independent blocks that can be solved concurrently, as shown in Fig. 1. This reformulation of the preconditioner does not impact number of iterations nor accuracy of the solution.

Shared memory parallelization of the SpMV kernel is straightforward when the matrix is stored in a compressed sparse row (CSR) format [15]. The kernel for a distributed CSR matrix starts with a local matrix-vector multiplication while initiating the point-to-point (P2P) MPI communication phase for collecting the interfaces from neighboring subdomains, then updates the solution with the received data. Therefore, there are three stages in this kernel: local SpMV, communication with neighbors, and solution update SpMV. The communication phase is typically done using non-blocking MPI calls that initiate the communication in advance which allows overlapping communication with computation; however, actual overlapping is hardware-dependent and not guaranteed [16]. In order to ensure asynchronous progress of the communication prior the MPI wait calls, we implemented a task-based hybrid MPI+OpenMP distributed SpMV. The main idea is to compute chunks of local matrix-vector multiply while an independent OpenMP task handles the communication progress. In this case, the hybrid task-based implementation forces the MPI runtime to progress in the non-blocking communication.

There are three variants of this linear solver code: MPI everywhere implementation with no overdecomposition of the linear system (MPI code), MPI+OpenMP implementation of the linear solver with the multithreaded overdecomposition of the system (OMP code), and MPI+OpenMP with taskbased implementation for the asynchronous progress of the P2P communication in the SpMV kernel (Task code). 


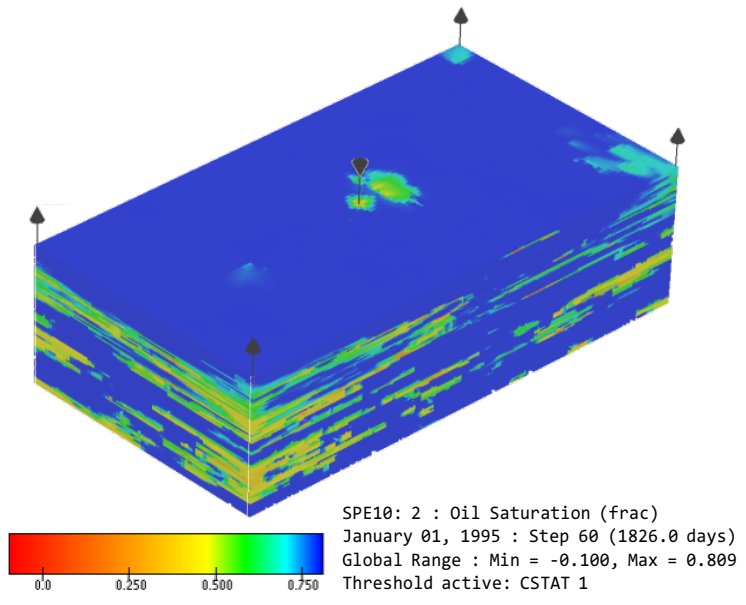

Fig. 2. Saturation profile obtained from SPE10 model

\section{PERFORMANCE OF MPI+OPENMP ON SPE10}

We test the performance of the hybrid approach using the SPE10 model, a heterogeneous standard reservoir model with a total of approximately 1 million $(\mathrm{M})$ cells $(60 \times 220 \times 85)$ [17]. The model consists of one water injector well at the center and four producing wells at the corners of the grid, as shown in Fig. 2. This modeled system is a two-phase (oil/water) problem. We used this model and derived models, which we generated by placing multiple SPE10 models next to each other with transmissibility barriers between them, effectively increasing number of grid blocks in the $X Y$ plane of the domain, for testing the performance of the hybrid approach in comparison with the MPI-everywhere approach. We test the performance of the formerly mentioned implementations on three Intel architectures: Haswell, Skylake, and Knights Landing (KNL). In all the test runs, we use the best hardware affinity pattern per code due to its high impact on the performance of both MPI and hybrid parallel models.

\section{A. Haswell Node}

The test runs in this section were performed on a node of Shaheen II, a Cray XC40 supercomputer at King Abdullah University of Science and Technology (KAUST). Shaheen consists of 6174 nodes of Intel Haswell (Xeon E5-2698v3), with $2 \mathrm{CPU}$ sockets per node, and 16 processor cores per socket. We used the SPE10 with $1 \mathrm{M}$ cells, and $2 \times 2$ and $4 \times 4$ times refined models, i.e., 1M, 4M, and 16M. Fig. 3 top graph, shows the time to solution of SPE10 models on the full node core count. The hybrid implementations are slightly faster and scaling better on the $4 \mathrm{M}$ and $16 \mathrm{M}$ models. The bottom graph shows the strong scalability for solving the SPE10 16M model. Fig. 4 shows the scalability of increasing number of threads per MPI execution of the Task implementation. The results of the strong scaling tests are shown in Fig. 4, where the product of the number of MPI processes and number of OpenMP threads are plotted against the time to solution of SpMV kernel. The legend shows the different colors for different number of MPI processes. OpenMP does not scale well across sockets, which
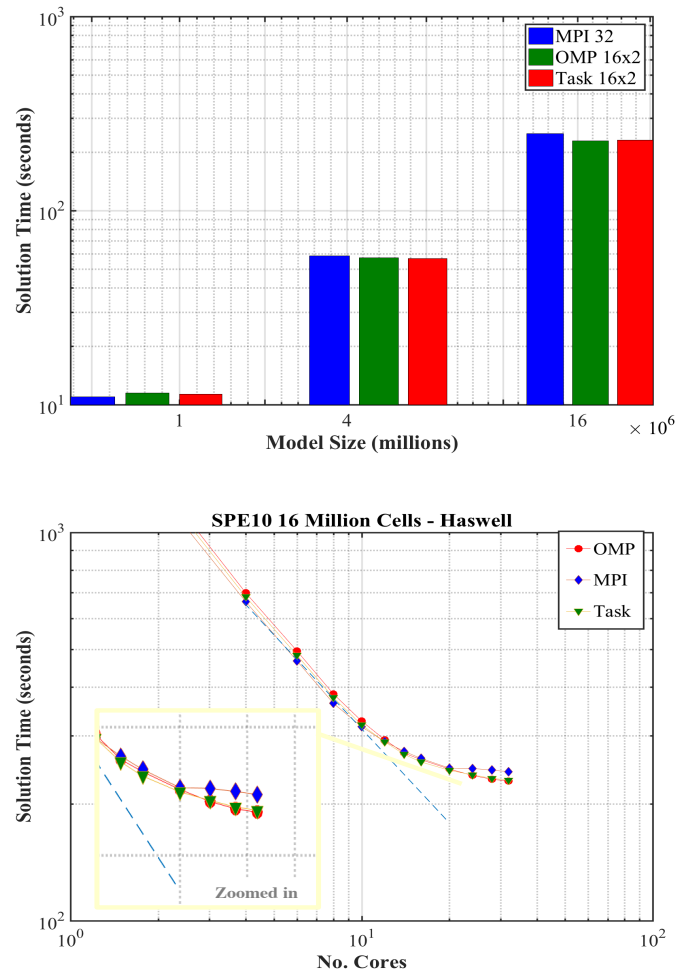

Fig. 3. The top figure shows the three implementations solving variants of SPE10 models using 32 of Haswell's available cores (no hyperthreading). The bottom one shows the scalability of the different implementations solving SPE10 16M cells model.

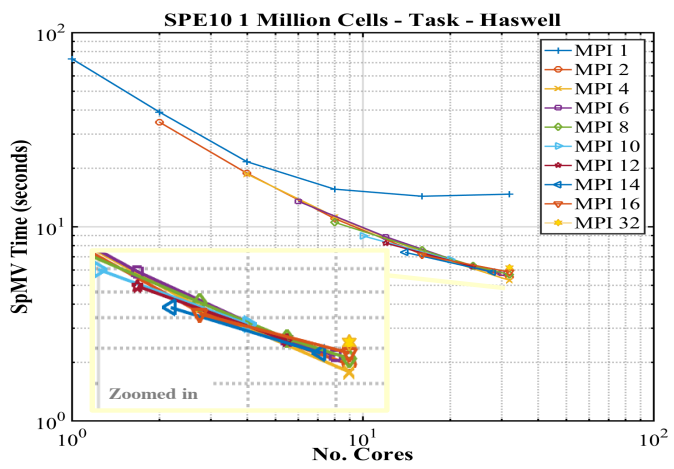

Fig. 4. The scalability of MPI+OpenMP with the task based implementation (Task) of SpMV kernel of the total solution time of SPE10 1M model on Haswell node.

is evident based on the results for OpenMP-everywhere (1 MPI, 32 threads). On the other hand, we can see that MPI alone does quite well if we follow the first point of each line. It gives the best results until 8 processes but is thereafter outperformed by the hybrid model $-4 \times 8$ MPI and OpenMP with taskification. Similar observation holds for OMP code where hybrid $2 \times 16 \mathrm{MPI}$ and classic OpenMP report the fastest time to solution of SpMV when using all 32 cores. We did not see significant differences in strong scaling between classic OpenMP and OpenMP with taskification. 


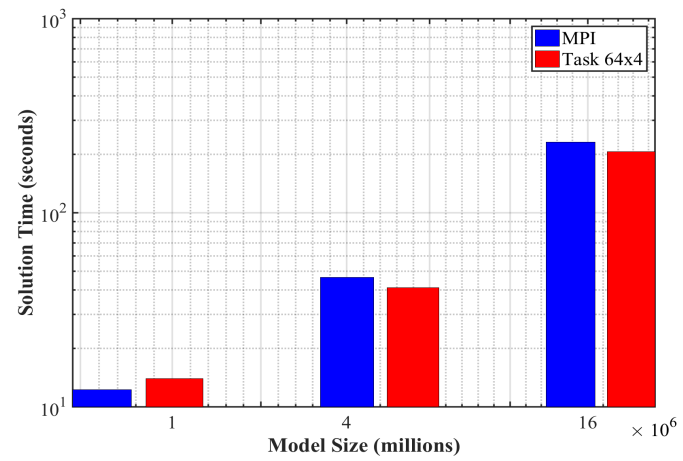

Fig. 5. Shows the hybrid and MPI solver implementations of SPE10 models on KNL node with hyperthreading. We report here the best time to solution for MPI Code using the full resources which is 64 cores.

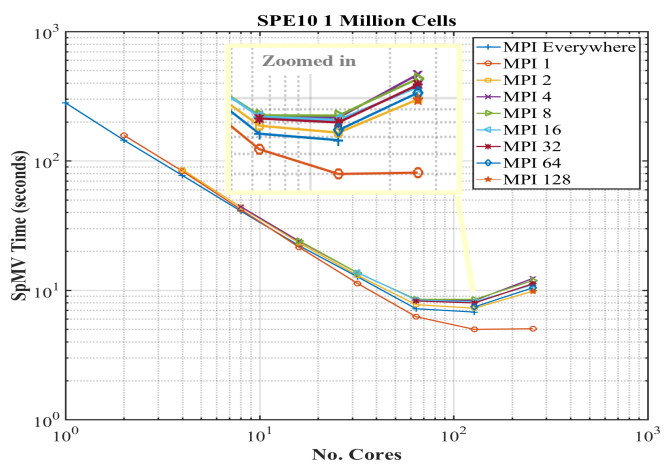

Fig. 6. Shows the scalability of both MPI and hybrid MPI+OpenMP taskbased implementation of SpMV kernel of the total solution time of SPE10 $1 \mathrm{M}$ model on $\mathrm{KNL}$ node.

\section{B. Knights Landing Node}

The test runs in this section were performed on Intel Xeon Phi (Knights Landing 7210), which is equipped with 64 hardware cores at $1.30 \mathrm{GHz}$ clock frequency [18]. KNL features different boot-time setups of the high bandwidth memory, MCDRAM, which can be configured as a third level cache, i.e., the cache mode, or as a NUMA node, i.e., the flat mode. The cache mode does not require any changes in the source code for using MCDRAM, whereas, in the flat mode the allocation of data in the MCDRAM is exposed at the code level. Therefore, the misses are more expensive in the cache mode compared to the flat mode.

We have tested all the possible combination of configurations, modes, and process allocations. Flat mode with explicit allocation of all the relevant solver arrays on MCDRAM with Quadrant clustering mode yielded the best performance for the reference implementations and we used this setting for all the reported results. The performance gain is $40 \%$ and $10 \%$ for the SpMV and total solution time respectively, as shown in Fig. 5 and Fig. 6. The latter figure shows all hybrid lines diverged at 128, whereas, OpenMP-everywhere scales up to 256 threads.

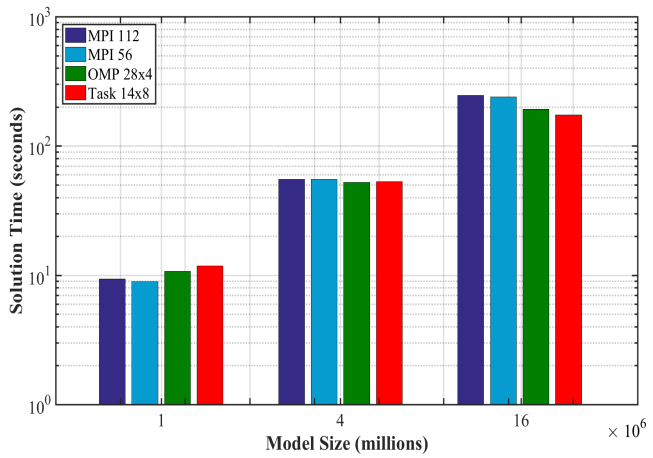

Fig. 7. The three implementations solving variants of SPE10 models using the full Skylake node available cores, i.e. 56 cores (112 threads with hyperthreading).

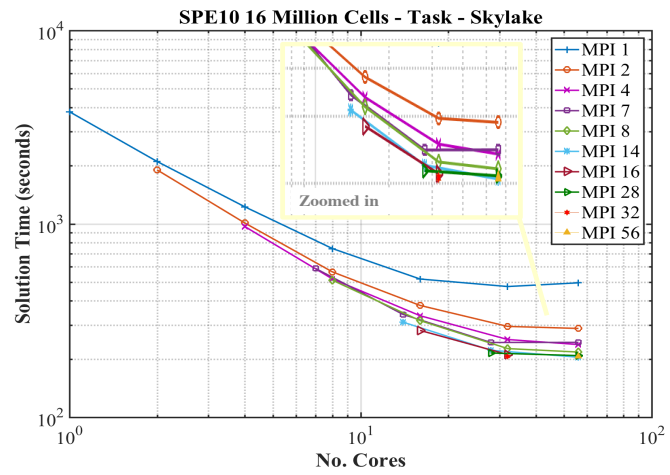

Fig. 8. The scalability of MPI+OpenMP task based implementation (Task) solving SPE10 16M model on Skylake node.

\section{Skylake Node}

In this section, we present the performance results on Skylake node (Xeon 8176), the successor of Haswell, with two sockets and 28 cores per socket, i.e., 56 hardware cores at 2.10 $\mathrm{GHz}$ clock frequency. The Skylake architecture has the best of both Haswell and KNL worlds. Its core is more complex with branch prediction similar to Haswell; and with vector processing unit similar to KNL, but runs at higher speeds than a KNL core. On this architecture, the hybrid implementations demonstrate good performance with almost 50\% speedup over MPI everywhere implementation on SPE10 16M model, as shown in Fig. 7. Figure 8 shows the strong scalability where the product of MPI processes and OpenMP threads are plotted against the time to solution on Skylake without hyperthreading. A common observation across architectures, OpenMP everywhere is not sufficient for scaling across sockets to the full core count, i.e., 56 cores in Skylake. In Fig. 8, hybrid $14 \times 4$ with taskification exhibits best time to solution.

\section{Across Architectures}

Figure 9 compares the performance of the discussed implementations on Haswell, Skylake, and KNL. Overall, multi- and many-core nodes expose multiple complexities at the level of the software and parallelization models. 


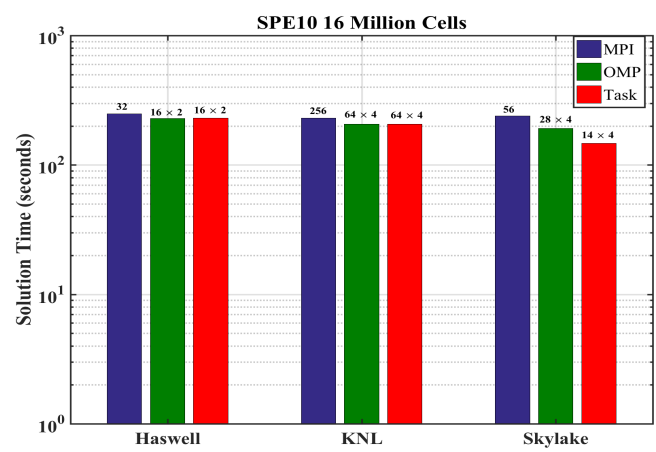

Fig. 9. The three implementations solving SPE10 16M cell model across architectures: Haswell, Skylake, and KNL.

Hyperthreading-enabled platforms favor the hybrid implementations MPI+OpenMP over the original one with MPI everywhere. On the other hand, MPI implementation show good scalability, especially on Haswell. The task-based hybrid code is generally faster than the OMP one. However, we expect the main benefits of the taskification are realized on a large distributed system due to the asynchronous progress of P2P communication. In the following section, we highlight the benefits of using hybrid on multiscale multiphysics real-world reservoir models.

\section{IMPACt on Production Reservoir Models}

In this section, we benchmarked 49 real-world reservoir models with a variety of characteristics, ranging from $49 \mathrm{~K}$ to $59 \mathrm{M}$ active cells on core counts from 4 to 3080 . The test runs were performed on Makman-2, a Dell PowerEdge R630 at Saudi Aramco. Makman-2 consists of 2 sockets of Intel Haswell, each socket has 12 cores at $2.5 \mathrm{GHz}$ (E5-2680v3). In this section, 4 processes with 6 threads each were used for all the hybrid runs. As shown on Fig. 10, top graph, the hybrid model (Task Code) reduces the total communication volume by half on average due to the reduction of number of subdomains per MPI. The main advantage of using the hybrid model is avoiding message passing communication within a node/NUMA, as shown in Fig. 10. Another advantage of hybridization is load balancing which minimizes number of edge-cuts without explicit data movement.

Fig. 10 bottom graph shows the ratio of MPI imbalance to Task code imbalance. Task code provides $90 \%$ reduction of imbalance on average. The imbalance ratio of each code has been computed with internal counter $\max \left(n_{i}^{\max }-n_{\text {balance }}, n_{\text {balance }}-n_{i}^{\min }\right) / n_{\text {balance }}$, where $n_{i}^{\max }$ is the maximum number of cells per subdomain, $n_{\text {balance }}$ is $\frac{\text { total number of cells }}{\text { number of subdomains }}$, and $n_{i}^{\text {min }}$ is the minimum number of cells per subdomain.

Figure 11 (top) shows the ratio of the maximum memory used per node (MPI/Task). Hybrid Task implementation offers more than $70 \%$ reduction of memory used per node. The bottom graph in Fig. 11 highlights the reduction in SpMV time
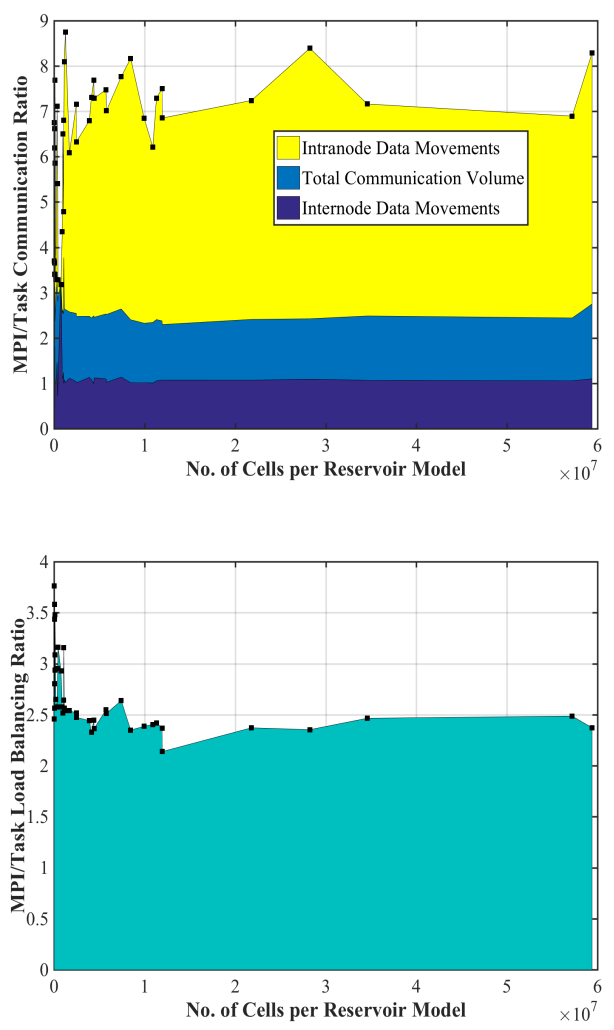

Fig. 10. Ratio of communication and load balancing of MPI to Task implementations solving different field cases; each black square corresponds to the number of cells of each field

due to P2P overlap with local computation using taskification as explained in Section III. On models with inter-node communication, Task implementation improves the performance of SpMV by a factor of $10-40 \%$. The speedup in time to solution of using the hybrid implementation over MPI everywhere one is up to $20 \%$, as shown in Fig. 12, but on average the performance is only slightly better. The main reduction in communication is for intranode data movements whereas for internode average of 5\% only. These results invite an obvious question of the impact of reducing the communication and load balancing benefits of the hybrid approach. The roofline model [19] shown on Figure 13 explains part of it. As the results indicate, the multithreaded SpMV and tridiagonal solve kernels are bandwidth limited at the L3 cache roof, whereas the SpMV of the pressure component is L2 cache limited. Hybridization helped move the bandwidth towards L3 cache roof however the code remains largely memory-bound.

\section{CONClusions AND Future Work}

We developed shared memory parallelization of the preconditioner and SpMV kernels of GigaPOWERS, Saudi Aramco's reservoir simulator, as complementary to the distributed memory MPI model. We discussed a comprehensive performance assessment of this approach on state-of-the-art many-core and multi-core Intel architectures using a variety of synthetic and real-world reservoir models. Our results indicate that hybrid 

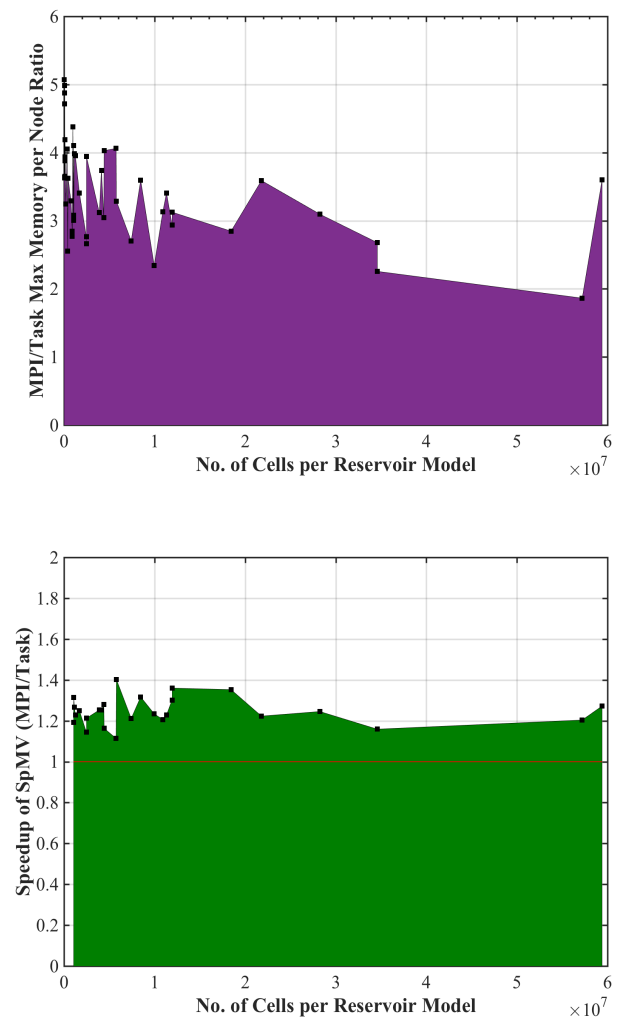

Fig. 11. Top graph is the ratio of maximum memory per compute node (2 sockets of Haswell) of MPI to Task implementations solving different field cases. Bottom graph is speedup of Task in SpMV of different field cases.

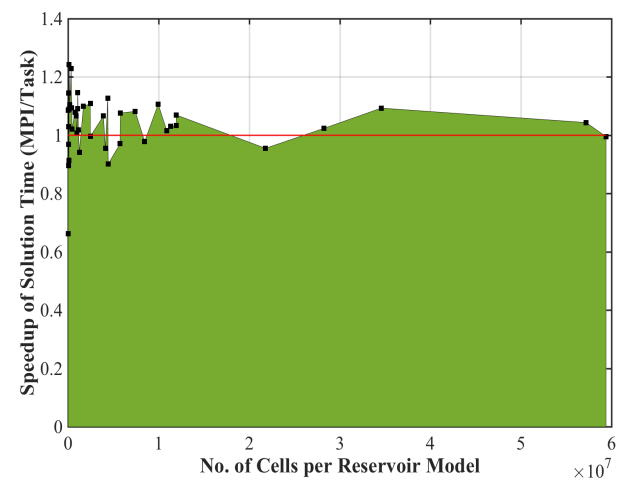

Fig. 12. Speedup of Task implementation in time to solution of different field cases.

model can outperform MPI-everywhere per-node on Intel Haswell, Knights Landing, and Skylake. The benefits of a hybrid model are the most pronounced on Skylake, where the performance boost can reach 50\% using 112 logical cores, which suggests the inevitability of hybridization for future hardware. Across all the configurations - MPI and various hybrid models - Skylake and KNL are slightly faster than Haswell. This work will be extended to consider further indepth analysis and profiling of the intranode strong scaling. Additionally, we will analyze the integration of the MPI layer

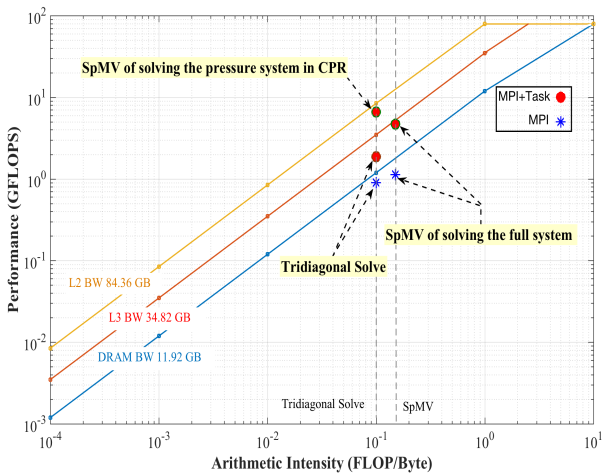

Fig. 13. Roofline model for a single node of Makman-2. The data was generated using Intel Advisor tool [20] tracing the multithreaded SpMV and Tridiagonal solve kernels.

in hybridization, where internode communication plays an essential role in performance.

The answer to whether hybrid MPI+OpenMP is worth the recoding effort is a complex one. Especially when porting production legacy code to the hybrid model, runtime reduction, reduced memory usage, improved load balancing, and internode traffic have to be weighed against developer effort and runtime complexities (from number of threads and multiple pinning strategies to number of blocks within MPI partition). The decision has to be on per-application basis and dependent on its particular domain decomposition, load balancing scheme, and the hardware in use. In our particular case, the improved load balancing and greatly reduced memory usage were even more significant than time to solution reduction.

\section{REFERENCES}

[1] R. Rabenseifner, G. Hager, G. Jost, and R. Keller, "Hybrid MPI and OpenMP Parallel Programming," in Recent Advances in Parallel Virtual Machine and Message Passing Interface, B. Mohr, J. L. Träff, J. Worringen, and J. Dongarra, Eds. Berlin, Heidelberg: Springer Berlin Heidelberg, 2006, pp. 11-11.

[2] L. Fung and A. Dogru, "Parallel Unstructured-Solver Methods for Simulation of Complex Giant Reservoirs," Society of Petroleum Engineers Journal, vol. 13, Dec. 2008.

[3] J. Wallis, "Incomplete Gaussian Elimination as a Preconditioning for Generalized Conjugate Gradient Acceleration," in SPE Reservoir Simulation Symposium. SPE, Nov. 1983.

[4] A. Behie and P. Vinsome, "Block Iterative Methods for Fully Implicit Reservoir Simulation," Society of Petroleum Engineers Journal, vol. 22, Oct. 1982

[5] "ECLIPSE. 2014. ECLIPSE Industry Reference Reservoir Simulator ," https://www.software.slb.com/products/eclipse/eclipse-2014.

[6] A. Dogru, L. Fung, U. Middya, T. Al-Shaalan, and J. Pita, "A NextGeneration Parallel Reservoir Simulator for Giant Reservoirs," in SPE Reservoir Simulation Symposium. SPE, Feb. 2009.

[7] H. Gahvari, W. Gropp, K. E. Jordan, M. Schulz, and U. M. Yang, "Modeling the Performance of an Algebraic Multigrid Cycle Using Hybrid MPI/OpenMP," in 2012 41st International Conference on Parallel Processing, Sept 2012, pp. 128-137.

[8] A. AlOnazi, G. S. Markomanolis, and D. Keyes, "Asynchronous Task-Based Parallelization of Algebraic Multigrid," in Proceedings of the Platform for Advanced Scientific Computing Conference, ser. PASC '17. New York, NY, USA: ACM, 2017, pp. 5:1-5:11. [Online]. Available: http://doi.acm.org/10.1145/3093172.3093230 
[9] L. Adhianto and B. Chapman, "Performance modeling of communication and computation in hybrid MPI and OpenMP applications," Simulation Modelling Practice and Theory, vol. 15, no. 4, pp. 481-491, 2007.

[10] T. Hoefler, J. Dinan, D. Buntinas, P. Balaji, B. Barrett, R. Brightwell, W. Gropp, V. Kale, and R. Thakur, "Mpi + mpi: A new hybrid approach to parallel programming with mpi plus shared memory," Computing (Vienna/New York), vol. 95, no. 12, pp. 1121-1136, 122013.

[11] V. Dzyuba, K. Bogachev, A. Bogaty, A. Lyapin, A. Mirgasimov, and A. Semenko, "Advances in Modeling of Giant Reservoirs," in Mathematical Methods in Fluid Dynamics and Simulation of Giant Oil and Gas Reservoirs. SPE, Jan. 2012.

[12] S. Jaure, A. Moncorge, and R. de Loubens, "Reservoir Simulation Prototyping Platform for High Performance Computing," in SPE Large Scale Computing and Big Data Challenges in Reservoir Simulation Conference and Exhibition. SPE, Sep. 2014.

[13] L. Fung, M. Sindi, and A. Dogru, "Multiparadigm Parallel Acceleration for Reservoir Simulation," Society of Petroleum Engineers Journal, vol. 19, Aug. 2014.

[14] H. Liu, K. Wang, Z. Chen, K. Jordan, J. Luo, and H. Deng, "A Parallel Framework for Reservoir Simulators on Distributed-memory Supercomputers," in SPE/IATMI Asia Pacific Oil and Gas Conference and Exhibition. SPE, Oct. 2015.

[15] G. Schubert, G. Hager, H. Fehske, and G. Wellein, "Parallel sparse matrix-vector multiplication as a test case for hybrid MPI+OpenMP programming," Computing Research Repository, vol. abs/1101.0091, 2011. [Online]. Available: http://arxiv.org/abs/1101.0091

[16] R. Brightwell and K. D. Underwood, "An analysis of the impact of mpi overlap and independent progress," in Proceedings of the 18th annual international conference on Supercomputing. ACM, 2004, pp. 298-305.

[17] M. Christie and M. Blunt, "Tenth SPE Comparative Solution Project: A Comparison of Upscaling Techniques," Society of Petroleum Engineers Journal, vol. 4, Aug. 2001.

[18] S. Ramos and T. Hoefler, "Capability Models for Manycore Memory Systems: A Case-Study with Xeon Phi KNL ," in Proceedings of the 31st IEEE International Parallel \& Distributed Processing Symposium (IPDPS'17). IEEE, May 2017.

[19] S. Williams, A. Waterman, and D. Patterson, "Roofline: An Insightful Visual Performance Model for Multicore Architectures," Commun. $A C M$, vol. 52, no. 4, pp. 65-76, Apr. 2009. [Online]. Available: http://doi.acm.org/10.1145/1498765.1498785

[20] "Intel Advisor," https://software.intel.com/en-us/advisor. 